\title{
LA CARACTERIZACIÓN ESTILÍSTICA DE LAS CLÁUSULAS ANÓMALAS EN EL HEXÁMETRO LATINO
}

M. ${ }^{8}$ Luisa Arribas

$U N E D$

I. En la frase «tout poète aime les mots en eux mêmes» ${ }^{1}$ encontramos una expresión que podría subscribir todo aquel que tuviera una cierta inclinación a la poesía. Debemos convenir, sin embargo, que, en el poema concreto, las palabras aisladas, por sí mismas, no alcanzan todo su valor; por el contrario, se hacen plenas cuando el poeta las integra en un contextofrase que debe articularse en función de la estructura métrica elegida.

Partiendo de estas premisas, y participando en la opinión más extendida, según la cual en la creación del hexámetro latino es fundamental la disposición tipológica de las palabras, vamos a analizar una serie de tipos clausulares que, al haber sido infrautilizados por los poetas de época clásica (seguramente por su especial disposición tipológica), han dado en denominarse "anómalos» o "excepcionales» por los especialistas en la materia.

Conocida la propensión de los poetas modélicos hacia los finales hexamétricos con la estructura $3+2$ (CONDERE GENTEM) y $2+3$ (CONDE SEPVLCRO) $o$, en su defecto, $2+1+2$ (GENTE TOT ANNOS $)^{2}$, debemos asimismo reconocer, como reverso de la medalla, la cautela de los poetas en relación al uso de otras distribuciones posibles. Este dato fue apuntado ya por Quintiliano cuando, en un pasaje de su Institutio oratoria $^{3}$, advierte que los finales del tipo FORTISSIMA TYNDARIDARVM, APPENNINOS, ARMAMENTIS, OREIONE, deben ser evi-

Herescu, N. I.: La poésie latine, Paris 1967, p. 73.

Nougaret, en su famosa Métrica, aporta cifras significativas en torno a la prepoderancia de los finales más regulares y a la restricción en el uso de los anómalos, si bien no tienen valor absoluto, dado que sus resultados proceden del examen de un número de versos demasiado reducido.

'Quintiliano: Inst, Or. IX. IV, 65. 
tados en el final de los versos, y que su presencia resulta "praemolle»: encontramos, pues, por primera vez el rechazo expreso de un tipo de cláusulas hexamétricas, y su vinculación a un matiz estilístico determinado.

Con respecto a las razones últimas que hayan podido guiar a los poetas en la elección de unos tipos de cláusulas frente a las otras posibles, se ha querido barajar, junto con la fuerza de la disposición tipológica de los términos, el hecho de la casi inexcusable coincidencia de acento de palabra y tiempo fuerte en las distribuciones acogidas como normales; sin embargo, éste no puede ser rasgo fundamental, ya que Virgilio es igualmente reticente al uso del tipo DI GENVERVNT (que provoca la homodinia) y GEMITV LACRIMISQVE (que la destruye), mientras que Ovidio, dentro de su parquedad, prefiere entre estos dos, el tipo con heterodinia.

Éste es problema que ha sido ya estudiado por nosotros, con todo detalle, en el trabajo que presentamos como Tesis Doctoral en febrero de $1988^{4}$. Los datos que con este motivo fuimos acumulando, han de servirnos ahora, en buena medida, para la caracterización estilística de cada una de estas cláusulas que solemos denominar «anómalas», y para definir su técnica de uso en los diversos géneros literarios.

1. Los primeros tipos clausulares a los que queremos referirnos son precisamente aquellos que hemos visto ya señalados por Quintiliano: en nuestra opinión, el autor alude concretamente a las cláusulas que se forman mediante un término pentasilábico o con un tetrasílabo de estructura dispondaica; es decir, aquellas formaciones en que los dos últimos pies se completan con un solo término; necesita, pues, consideración aparte el caso de los tetrasilabos finales con estructura de jónico «a minore», que deben ayudarse de un monosílabo o de la última sílaba de un polisílabo precedente para rellenar la cláusula.

En la primera época del hexámetro, la que podríamos llamar de introducción -donde debemos considerar las obras de Ennio, Lucilio y Lucrecio, por el carácter más arcaico de sus versos-, ambos tipos clausulares presentan desigual fortuna: el cuadro que puede resumir el comportamiento de estos poetas es el siguiente:

\begin{tabular}{|c|c|c|c|}
\hline & ENNIO & LUCILIO & LUCRECIO \\
\hline VADRVPEDANTVM & $\begin{array}{c}21 \text { ej. } \\
3.65 \%\end{array}$ & $\begin{array}{r}19 \mathrm{cj} . \\
4.08 \% "\end{array}$ & $\begin{array}{l}306 \mathrm{ej} . \\
4.12 \%\end{array}$ \\
\hline
\end{tabular}

- Arribas, M. L.: Estudio métrico acerca de la cláusula tipo ARMAQVE NOSTRA en el hexámetro dactilico. Madrid 1988. 


\begin{tabular}{|c|c|c|c|}
\hline & FNNIO & LUCILIO & LUCRECIO \\
\hline APPENNINOS & $\begin{array}{l}4 \text { (1) } \\
\text { (1) }\end{array}$ & - & $\begin{array}{c}28 \mathrm{cj} \\
0.37 \%\end{array}$ \\
\hline
\end{tabular}

Evidentemente, el tipo pentasilábico fue acogido con generosidad, primero por Ennio y luego por el satírico y el filósofo.

El pentasilabo utilizado por Ennio no muestra ninguna caracterización especial, si acaso suele acoger con frecuencia sustantivos compuestos del tipo ALTIVOLANTVM $(A n$. I, 85) o BELLIGERANTES (An. VI, 187), pero admite igualmente nombres propios: AEACIDARVM $(A n . \mathrm{V}, 174) \mathrm{o}$ FVFETIOEO $(A n$. II, 139) y formas verbales: INCREPVISTI $(A n . \mathrm{I}, 104)$ o SOLLICITABANT (An. XVI, 416).

Para la gustosa aceptación de este tipo por parte de Ennio parece razón principal los estrechos lazos con los que el poeta se sentía vinculado a las formas homéricas ${ }^{5}$, dado que en la Ilíada y la Odisea son muy frecuentes los finales de estas dimensiones. Esta disposición es utilizada en proporción aún mayor por Lucilio y Lucrecio. El satírico, posiblemente, porque, a pesar de haber introducido algunas variaciones con vistas sobre todo a aproximar su hexámetro a la lengua hablada, sigue considerando como modelos a Homero y a su más directo predecesor Ennio.

Con respecto a Lucrecio, este tipo de finales - además de estar recomendado por el uso de Ennio y los modelos griegos-- debió de resultarle especialmente cómodo, porque puede recoger vocablos de amplio volumen fónico que eran esenciales en su exposición: apoya esta idea el hecho de que la frecuencia de estos términos varía de acuerdo con el tema que el poeta está considerando en cada ocasión: así, MATERIAI se repite en final de verso en 12 ocasiones en el libro I, y en 22 en el libro II; y PRINCIPIORVM ocupa la cláusula cinco veces en el libro I y 22 en el II, disminuyéndose su porcentaje en los restantes libros. La razón, según aduce Bailey, estaría en que Lucrecio en los dos primeros libros expone la constitución de la materia y la naturaleza de los átomos, por lo que, con extraordinaria frecuencia, debe recurrir a estos términos, necesidad que decrece en los siguientes, al haber cambiado el tema sobre el que se realiza la exposición.

Sin embargo, aparte de por la conveniencia de lenguaje y métrica, parece que Lucrecio se dejó guiar para la elección de estos finales por consideraciones de tipo estilístico, estimándolos más aptos para elevar o hacer más

'Cupaiuolo, F.: Un capitolo sull'esametro latino, Nápoles 1963, p. 11.

' Lucretius: De rerum natura libri sex I, ed. C. Bailey, Oxford 1943, p. 117. 
notable un contenido, ya que en el remate de algunos libros esta distribución se repite: asi ocurre, por ejemplo, en el libro VI, donde utiliza un pentasílabo final en los versos $1260,1263,1275,1277$ y 1286, en opinión de Bailey «to give a especial dignity to the conclusion of the poem», opinion que por nuestra parte subscribimos.

No debió de resultar, sin embargo, tan fácil o tan grato el uso de los finales dispondaicos a la manera homérica: es decir, reuniendo los semipiés décimo y undécimo en un sólo término, generalmente tetrasilábico.

Mientras que en Homero la proporción de los versos espondaicos asciende al 5,5 \% alcanzan sólo un $0,69 \%$. Incluso si consideramos aquellos once versos que presentan espondeo en el quinto pie, pero cuya realización no coincide con la ortodoxa griega, la proporción en los Anales no supera el $2 \%$, quedando, pues, muy lejos de su modelo.

Cabría pensar en una supuesta escasez de términos con estas caracteristicas. Ciertamente los tetrasílabos con moloso inicial no son muy frecuentes en la lengua latina, pero más restringidos aún parecen los que presentan la estructura $u v-x$ y. sin embargo, fueron bien acogidos ${ }^{8}$.

Más radical, sin duda, resulta el proceder de Lucilio que prescinde absolutamente del tetrasílabo espondaico. Es posible que el satírico recibiera este tipo de cláusulas como característico de la poesía alejandrina y, conscientemente, quisiera marcar distancias; o quizá las palabras de este tipo le resultaran - como después a Quintiliano- «praemollia»y, por tanto, poco adecuadas para la expresión satírica.

En Lucrecio hay también una clara disminución respecto al uso de esta cláusula. No se percibe en el filósofo una especialización del tipo, aunque en un alto porcentaje se ocupa con formas verbales, sean infinitivos o formas conjugadas, apareciendo de manera más esporádica algunos sustantivos, como INTERVALLVM, en IV, 187 o SPICARVMQVE, en III, 198.

Si en los poetas que presentan los rasgos más arcaicos la cláusula constituida con un pentasílabo era objeto de predilección, y los finales espondaicos perecían rechazados, en los que se sitúan en la época de expansión del hexámetro o en transcición hacia las formas clásicas, encontramos una clara evolución.

Las cifras que hemos podido recoger en nuestro estudio son las siguientes:

Cordier, A.: Les débuts de l'hexamètre latin. Ennius, París, p. 34.

"Según Nougaret en su Analyse verbale comparée du De Signis et des Bucoliques, París 1966. pp. 41-42, Cicerón emplea en De Signis 76 vocablos con la estructura $-\ldots-\mathrm{x}$, y sólo 30 con la disposición $v e-x$. 


\begin{tabular}{ccc} 
& CATULO & CICERÓN \\
\cline { 2 - 3 } QVADRVPEDANTVM $\ldots \ldots \ldots \ldots . . .$. & $6,75 \%$ & 6 \\
& 32 & $0,81 \%$ \\
APPENNINOS $\ldots \ldots \ldots \ldots \ldots \ldots \ldots \ldots$ & $4,03 \%$ & $0,14 \%$
\end{tabular}

La primera cuestión que merece la pena considerar es la renuencia de estos autores a cerrar el hexámero con una forma pentasilábica: su proporción de uso, que rondaba el $4 \%$, en los poetas de rasgos arcaicos, ha descendido por debajo del $1 \%$. Aunque los porcentajes sean semejantes en ambos autores, su forma de empleo no lo es, ya que, mientras Catulo inserta cualquier tipo de vocablo con estas características ${ }^{9}$, Cicerón se decanta hacia los nombres propios, o en todo caso palabras de carácter descriptivo ${ }^{10}$.

Si en Catulo este tipo de finales parece simplemente memoria de una cláusula que tiende a desaparecer, en Cicerón ha quedado ya seleccionada con unas matizaciones estilísticas claras, entre las que podemos señalar el deseo de prestar al verso un cierto tono helénico, ya que de entre los seis ejemplos recogidos, tres están ocupados por un término griego.

Por el contrario, con respecto a la cláusula ocupada por un vocablo de estructura dispondaica, la matización viene de la mano de Catulo. El poeta, que se distingue sobre todo - como afirma Cupaiuolo" en su atinado trabajo en torno al hexámetro de este poeta- por su deseo de imitar el verso utilizado por los poetas alejandrinos, siente una especial predilección por el espondeo en el pie quinto: que tal preferencia se vincula de manera casi exclusiva a la técnica alejandrina lo sostiene el hecho de que este tipo de finales aparece en gran número en los poemas que siguen la técnica del Epylion y la práctica neotérica también en otros aspectos - como ha determinado J. Ross ${ }^{12}$-, mientras que en los epigramas, que heredan una tradición más antigua, cercana a la práctica de Ennio, el porcentaje de los

- En Catulo registramos los siguientes: EXILVERE $(62,8)$; EGREDIENTEM $(64,114)$; ALITIBVSQVE (64,152); CONTREMVERVNT (64,205); LAVDAMIA (68b,105); SESQVIPEDALIS $(97,5)$.

${ }_{10}$ Cf. The poems of Cicero, de W. W. Ewbank, publicado en Nueva York y Londres, 1978.

Como él recogemos los finales ANGVITENENTIS (Arat. XIII, 1 y XXXIII, 293); CASSIEPIA (Arat. XXXIII,1); CASSIEPIAE (Arat. XXIII, 23); TAYGETEQVE (Arat. XXXIII, 35) y POSTERIORES (Arat. XXXIII, 358).

La edición consultada por nosotros, y a la que pertenecen las referencias de los versos citados, es la publicada por J. Soubiran en París 1972.

"Cupaiuolo, F.: Studi sull' esametro di Catullo, Nápoles 1965, p. 13.

12 Así lo propone D. O. Ross. fijándose en variados aspectos de la composición del verso catuliano y haciendo especial hincapié en el uso de la elisión dentro del conjunto de la obra del poeta de Verona. En Style and tradition in Catullus, Cambridge 1969, pp. 115-136. 
finales dispondaicos no alcanza el $2 \%$ (frente a casi un $5 \%$ en los poemas de corte alejandrino).

Para Cicerón, en cambio, el final dispondaico parece una muestra de mal gusto propia de los "neoteroi», contra los que no duda en lanzar sus invectivas y contra los que, a modo de burla, compone un hexámetro que envía a su hermano Atico ${ }^{13}$.

En su obra poética, concretamente, sólo aparece un ejemplo (ORIONIS, Arat. XXXIII, 3), que pudo ser admitido en razón de la presión especial que ejercieron los nombres propios en el esquema métrico.

Con respecto a los poetas de época augústea la situación es la siguiente:

\begin{tabular}{|c|c|c|c|c|c|}
\hline & VIRGILIO & HORACIO & TIBULO & PROPERCIO & OVIDIO \\
\hline QVADRVPEDANTVM & $\begin{array}{c}21 \\
0,16 \%\end{array}$ & $\begin{array}{c}27 \\
0,64 \%\end{array}$ & - & $\stackrel{2}{0,10 \%}$ & $2.2 \%$ \\
\hline PPENNINOS & $\begin{array}{c}16 \\
0,12 \%\end{array}$ & $\begin{array}{c}5 \\
0,11 \%\end{array}$ & - & $\begin{array}{c}4 \\
0,20 \%\end{array}$ & $\begin{array}{c}36 \\
0,15 \%\end{array}$ \\
\hline
\end{tabular}

La primera consideración que salta a la vista es la absoluta renuencia de Tibulo hacia estos tipos clausulares. Bien podríamos decir que su comportamiento supone una ruptura total con respecto a los moldes anteriores y contemporáneos ${ }^{14}$.

Con todo, hay que reconocer que los porcentajes de uso han decrecido notablemente en relación a los registrados en la obra de los poetas antiguos.

Si nos acercamos a Virgilio como primer poeta —desde el punto de vista cronológico, y desde el punto de vista de la técnica creadora- observamos que, con respecto al tipo pentasilábico, su opción ha quedado claramente determinada en las Bucólicas y en las Geórgicas: todos los nombres con esta disposición son propios o helénicos; en la Eneida casi todos presentan ese mismo carácter: las únicas excepciones las tenemos en QVADRVPEDANTVM, - vocablo eminentemente expresivo y con antecedente en el mismo Ennio- y en ANCIPITEMQVE - término muy descriptivo que con su amplitud evoca la enorme dificultad del laberinto de Creta. Es la misma técnica de Cicerón que, de alguna manera, se va a «institucionalizar» por la elección de Virgilio, según percibimos en las obras de Propercio y Ovidio.

Propercio realiza sus cláusulas pentasilábicas con el nombre HIPPODAMIAE (I, VIII, 35) - propio y helénico- y con la forma verbal INCREPITARENT, integrada en un dístico de claras reminiscencias griegas,

1 "Cicerón: ad At. 7, 2, 1 .

14 Esta es la actitud que se repite en el Corpus Tibullianum en relación a la mayoría de las cláusulas denominadas anómalas. 
por el apelativo Nereides en el hexámetro y los nombres Nesaee y Cymothoe en el pentámetro.

En Ovidio encontramos un solo ejemplo en los Fastos (IV, 475) y otro en las Metamorfosis (XII, 441): en ambos casos los términos son nombres propios de ciudades - TAVROMENVMQVE y TELEBOASQVE, respectivamente-, añadiendo el segundo a esta caracteristica su corte helénico.

Con respecto a Horacio, el tipo con pentasílabos aparece en las Sátiras con una proporción del $0,85 \%$, y en las Epistolas con sólo un $0,45 \%$. Entre ellos, algo menos de la mitad $(42,6 \%)$ está representada por nombres propios y un número muy reducido recoge términos helénicos.

Por el comportamiento de Horacio -las proporciones de este tipo, más elevada en las Sátiras; la diversidad de los términos elegidos; la mínima evocación de los modelos griegos- suponemos que el autor se ha situado, voluntariamente, más cerca de los poetas arcaicos - especialmente de Lucilio- que de su contemporáneo Virgilio.

En relación con la cláusula dispondaica, se hace bien patente el influjo de su antecesor Lucilio, pues en las Sátiras prescinde absolutamente de este tipo, mientras que en las Odas, Épodos y Epistolas -cuya factura sigue más de cerca la dẻl verso heroico-, aparece recogida en algunas ocasiones. En los cinco ejemplos registrados por nosotros, de acuerdo con la técnica virgiliana, aparecen los nombres propios ORIONIS ( $O d$. I, 28,21), CYLLENEA (Epod. 13,9), PHOCAEORVM (Epod. 16,17) y APPENNINOS (Epod. 16,29), tres de ellos con incuestionable raigambre helénica. La única excepción la tenemos en ACCIDENTI $(A . P .467)$ en una línea que ha querido impugnarse por Ribbeck y Müller ${ }^{15}$, precisamente por tratarse del único verso espondaico con estas características.

Parece, pues, evidente que, partiendo de una suficiente representación en los primeros poetas - a la manera de los modelos griegos- la cláusula tipo QVADRVPEDANTVM ha quedado reservada para servir como instrumento de la técnica helenizante o, en todo caso, para recoger términos de gran expresividad. Con condiciones parecidas a las de los poetas de la primera época y con un porcentaje habitualmente inferior al de los modelos griegos, la cláusula tipo APPENNINOS ha servido en época augústea para la evocación de la técnica neotérica, que los autores han apoyado, en buena medida, con el empleo de términos helénicos.

Las únicas excepciones estarían en la obra de Tibulo -que no muestra mayor interés por las cláusulas anómalas - y de Horacio satírico, cuyo modelo, por encima de todo, está en Lucilio.

15 Brick, C. O.: Horace on poetry, Cambridge 1971, p. 428. 
2. Otro tipo de finales minoritario en época augústea es el constituido por un término en figura de jónico «a minore». Para completar la cláusula debe precederse de un monosílabo largo o de la sílaba larga final de un polisílabo. En el primer caso hemos de contar siempre con una cláusula homodínica, en el segundo, sólo cuando la silaba que ocupa el V longum procede de un polisílabo que ha elidido su último elemento.

El cuadro que representa los versos de este tipo clausular en la primera época es el siguiente:

\begin{tabular}{|c|c|c|c|}
\hline & ENNIO & LUCILIO & LUCRECIO \\
\hline DI GENVERVNT & $\begin{array}{c}16 \\
2,78 \%\end{array}$ & $\begin{array}{l}14 \\
3 \%\end{array}$ & $\begin{array}{c}154 \\
2,07 \%\end{array}$ \\
\hline GEMITV LACRIMISQVE .................. & $\begin{array}{c}12 \\
2,08 \%\end{array}$ & $\begin{array}{c}3 \\
0,64 \%\end{array}$ & $\begin{array}{c}13 \\
0,17 \%\end{array}$ \\
\hline
\end{tabular}

Ennio, en sus Anales, utiliza casi por igual la distribución homodínica y la heterodínica, y sus cláusulas, que no muestran preferencia hacia un tipo de vocablos en particular, parecen ser la simple transposición a la lengua del Lacio de uno de los finales utilizados frecuentemente por Homero.

En Lucilio, sin embargo, hay un predominio clarísimo de las cláusulas de carácter homodínico: cabría, por tanto, pensar que el poeta sentía preferencia por aquellos finales que ofrecían coincidencia de acento de palabra y tiempo fuerte, al menos en esta distribución concreta.

Entre los 17 vocablos con figura de jónico «a minore» situados en el broche del verso, cinco pertenecen al acervo griego: resulta curioso comprobar que dos de ellos ${ }^{16}$ se construyen con heterodinia (lo que representa un $66,66 \%$ sobre el total en el tipo GEMITV LACRIMISQVE) y los otros tres con construcción homodínica (con sólo un $21,42 \%$ sobre el total en el tipo DI GENVERVNT). Interpretando estos datos podríamos afirmar que Lucilio prefiere hacer coincidir el final heterodínico con la evocación helénica, quizá porque la homodinia era ya considerada como un fenómeno específico dentro de la creación poética latina.

En Lucrecio podemos encontrar igualmente la tendencia a configurar estas cláusulas con ayuda de la homodinia. La elevada proporción de este tipo - además de suponer la continuidad con respecto a la línea de Lucilioviene favorecida por el uso del esquema repetitivo del que tan frecuentemente hace uso el autor. Así, según nuestros estudios ${ }^{17}$, el término ANIMAI aparece ocupando este lugar en 25 versos (de los 31 en que Lucrecio lo utiliza), y el vocablo ANIMANTES se aplica en 30 ocasiones.

\footnotetext{
16 TYTYPHANTAS, en Sát. I, 13 y ALOCHOEO, en Sát. 1, 22,2.

1 Datos recogidos en Roberts, L.: A concordance of Lucretius, Nueva York, 1977.
} 
Con todo, no se detectan en el autor preferencias marcadas hacia un determinado tipo de palabras: en general Lucrecio, para rellenar esta cláusula, utiliza formas verbales o términos que son importantes en su exposición filosófica, intentando que queden en relevancia.

En la época de transición la frecuencia de estos tipos es la siguiente:

\begin{tabular}{|c|c|c|}
\hline & CATULO & CICERÓN \\
\hline DI GENVERVNT & $\stackrel{9}{1,13 \%}$ & $\begin{aligned} 3 \\
0,41 \%\end{aligned}$ \\
\hline GEMITV LACRIMISQVE ...................... & $\begin{array}{r}5 \\
0,63 \%\end{array}$ & - \\
\hline
\end{tabular}

Catulo y Cicerón son autores claramente inclinados a construir estos finales con realización homodínica. En Catulo, por otra parte, la evocación de los modelos griegos es evidente, de acuerdo con las tres razones que expondremos a continuación:

1. utiliza solamente vocablos de origen griego, concretamente HYMENAEVS y CALATHISCI;

2. hace uso repetido del hiato en la secuencia O HYMENAEE, fenómeno propio de la técnica helenizante, y mal aceptado según la normativa de la poesía romana;

3. Catulo no hace uso de este final en sus epigramas, herederos - como ya quedó señalado - de la tradición más puramente latina: sus ejemplos los encontramos en los poemas de corte neotérico.

En cuanto a Cicerón, entendemos que se ha acercado a este tipo de cláusula con especial circunspección, ya que sólo hemos podido recoger en su obra tres ejemplos, todos ocupados por un nombre propio $\mathrm{y}$, en este caso, de origen latino. Sistemáticamente, el autor ha elegido la realización homodínica.

El rétor-poeta, pues, restringe el uso de estas cláusulas, haciéndolas aptas exclusivamente para poner en relevancia nombres propios, sin haber querido vincular este tipo a la técnica helenizante: en ese sentido resulta más adecuada la realización homodínica que ha preferido utilizar.

En los autores de época augústea la situación es la siguiente: 
VIRGILIO HORACIO TIBULO PROPERCIO OVIDIO

\begin{tabular}{|c|c|c|c|c|c|}
\hline DI GENVERVNT …….... & $\begin{array}{c}24 \\
0,18 \%\end{array}$ & $\begin{array}{c}25 \\
0,50 \%\end{array}$ & - & $\begin{array}{c}1 \\
0,05 \%\end{array}$ & - \\
\hline $\begin{array}{l}\text { GEMITV } \\
\text { LACRIMISQVE. }\end{array}$ & $\begin{array}{c}27 \\
0,20 \%\end{array}$ & $\begin{array}{c}11 \\
0,26 \%\end{array}$ & - & $\begin{array}{c}2 \\
0,10 \%\end{array}$ & $\begin{array}{c}12 \\
0,05 \%\end{array}$ \\
\hline
\end{tabular}

Virgilio utiliza este tipo de cláusulas tanto con realización homodínica como heterodínica - en todo caso esta última supera ligeramente la proporción de su contraria-. Entre los 51 términos que aparecen con esta distribución sólo cuatro tienen un carácter netamente latino (COMITATV, ANIMVMQVE, LACRIMISQVE y VLVLATV), siendo los demás de origen helénico. Este solo hecho podria habernos hecho pensar que Virgilio ha elegido este tipo de finales para la evocación más directa de los modelos griegos, pero nuestra afirmación se refuerza por el dato de que, junto a la casi habitual transcripción de términos helénicos en esta posición, se detectan fenómenos como el alargamiento en "arsis», la admisión del hiato o el mantenimiento de vocal larga ante inicial vocálica subsecuente.

Las cuatro cláusulas, ya señaladas, que presentan un término de indole latina, aparecen marcadas en cada caso por matizaciones estilísticas ${ }^{18}$; en ninguna de ellas encontramos un final de carácter anodino. En consecuencia, podríamos decir que este tipo ha quedado determinado en Virgilio con rasgos muy específicos que lo presentan como clara manifestación de la técnica helenizante.

Horacio, por su parte, da muestras de conocer esta caracterización «a la griega» de las cláusulas con jónico "a minore», pero la utiliza en contadas ocasiones: nombres helénicos aparecen en Od. I, 7, 1, (MYTILENEN), Ep. II, 1, 58, (EPICHARMI), A.P. 355 (CITHAROEDIS) y Sat. I, 7, 16, (DIOMEDI).

La proporción -como es fácilmente comprensible-, siendo mínima, resulta mucho más abultada en el hexámetro lírico y en el de las Epistolas, pues en los 2113 versos de las Sátiras sólo aparece un término griego entre los 23 recogidos $(4,34 \%)$, mientras que entre los 13 representantes de la poesía no satírica, se registran tres helenismos $(23,07 \%)$.

De nuevo Horacio marca sus diferencias en el uso de las cláusulas anómalas, según las utilice para la sátira o para los otros géneros poéticos.

Tibulo, también en esta ocasión, ha querido prescindir de esta fórmula no regular, siguiendo la línea que ya se vio esbozada en los estudios de las cláusulas anteriores.

Propercio, en su obra, se muestra seguidor de la técnica virgiliana, pues,

${ }^{18}$ Cf. Arribas, M. L.: O.c. p. 231. 
aunque su proporción de uso es inferior a la del mantuano, los tres casos en que acoge esta distribución la realizan mediante términos griegos: ACHELOI (II, XXXIV, 33), TEREBINTHO (III, VII, 49) y HYACINTHOS (IV, VII, 23). En dos casos se realizan con heterodinia y en uno existe coincidencia de acento de palabra y tiempo fuerte.

Con respecto a Ovidio, aunque el uso de este tipo parece aún más restringido, continúa en su trayectoria la línea dibujada por Virgilio, pues los ejemplos recogidos en las Metamorfosis y el Ars amandi utilizan siempre términos helénicos y aceptan además el hiato y el mantenimiento de la cantidad larga de una vocal ante la inicial subsecuente.

La limitación que el poeta se impone, tocante a no utilizar este tipo de finales con realización homodínica, quizá podría entenderse como un deseo de aproximación a los modelos griegos, ya que, como sospechamos en Lucilio y Cicerón, la caracterización de la cláusula por la homodinia podía quizá entenderse como creación típicamente romana —dada la absoluta inoperancia de esta particularidad entre los poetas griegos-. En este sentido, Ovidio habría buscado un punto más en la condición helenizante de este tipo clausular.

3. Otro final acogido con generosidad por Ennio y fácilmente interpretable como clara transposición del modelo homérico es el constituido por un monosílabo final aislado, figura que en el autor de los Anales alcanza casi el $6 \%$. En el amplio número de ejemplos recogidos -32 en 565 versos- no se aprecia una intención estilística clara, pues, aunque sitúa con mayor frecuencia términos que podrían considerarse relevantes desde el punto de vista semántico, como LVX $(A n$. I, 94) o SOL $(A n$. I, 96), aparecen también formas verbales (SVNT, $A n$. I, 106), pronominales (SOS, $A n$. VII, 244) o conjuntivas (SIC, An. XIII, 381).

Hasta cierto punto significativa es la cláusula monosilábica en Lucilio, pues, de los 19 casos registrados, sólo cinco se ocupan con un término semánticamente pleno, siendo los otros 14 formas gramaticales o del verbo copulativo. Con ello, el satírico intentaba seguramente quitar solemnidad al final del hexámetro, y acercarlo al lenguaje conversacional. La frecuencia de este tipo $(4,08 \%)$ parece también representativa, pues, junto con la fórmula QVADRVPEDANTVM es cláusula preferida entre las anómalas, como clara prolongación del status homérico.

Lucrecio ofrece ya una proporción de uso más reducida en este tipo de finales, dado que su promedio sólo asciende al 2,5\%.

El conjunto de sus términos ofrece una cierta homogeneidad, pues aproximadamente la mitad de ellos se ocupa por monosílabos de carácter gramatical y la otra mitad por vocablos que convienen especialmente a su discusión filosófica: así, entre los 92 términos monosilábicos que aparecen 
como broche en los tres primeros libros del De rerum natura, 19 veces encontramos la palabra RES; 13, VIS y 7 MENS.

Permanece, pues, Lucrecio en la línea que combina la continuidad en los usos de sus modelos y la salvaguarda de la exposición filosófica, que promueve el esquema repetitivo y la ocupación de la cláusula por términos importantes en su discusión.

En la época de transición del hexámetro, la proporción de uso del tipo con monosilabo final es la siguiente:

\begin{tabular}{|c|c|c|}
\hline & CATULO & CICERÓN \\
\hline EXORITVR SOL & $\begin{array}{c}8 \\
1 \%\end{array}$ & $\begin{array}{r}6 \\
0,81 \%\end{array}$ \\
\hline
\end{tabular}

En cualquiera de los dos autores se puede percibir una fuerte recesión con respecto a los porcentajes de sus antecesores: debemos comprobar si tal comportamiento se debe a una mera inapetencia hacia este tipo de términos o a una clara especialización de esta disposición clausular.

Según los datos que en nuestro estudio hemos reunido, Catulo utiliza preferentemente formas pronominales (3 ME, $1 \mathrm{TE}, 1 \mathrm{QVI})$, frente a un sustantivo con sentido pleno, MORS, y dos formas del verbo copulativo (EST y SIT) ${ }^{19}$. Hay que reconocer, sin embargo, que las formas pronominales pueden a veces recoger una gran dosis de expresividad como en 107, 5 , donde TE se refiere a Lesbia; en 66, 63 y 66, 91 donde ME representa al mismo rizo de Berenice, y en 68, 33 - con la secuencia APVD ME - en que el propio Catulo pone de relieve sus carencias para así quedar exculpado ante su amigo Manlio.

Cicerón, por el contrario, elige sistemáticamente sustantivo de carácter casi técnico en Aratea: I PES, 3 NOX, 1 SOL, 1 VIS ${ }^{20}$. En este último caso, concretamente, se da la circunstancia de que la secuencia EQVI VIS es evocación del final $\mathfrak{\eta} \varepsilon \lambda$ iov ís de Aratos, $P h ., 286$. Como dato curioso hemos de reseñar que los términos monosilábicos de carácter gramatical suelen aparecer precedidos de otro monosílabo con el que forman "mot-métrique», originando una cláusula similar a las acostumbradas de final bisilábico: HAC EST (VII, 4); EX SE (XXXIII, 153); PRAE SE (XXXIII, 429).

Con ello, el uso estilizado de Cicerón y su deseo de proponer una cláu-

${ }^{19}$ Los lugares en que aparecen los citados monosílabos son: $\mathrm{ME}(66,63 ; 66,91 ; 68,33)$ ' MORS $(68,19)$; TE $(107,5)$; QVI $(112,1)$; EST $(62,45)$; SIT $(115,3)$.

${ }_{20}$ Los lugares en que aparecen los monosílabos citados son: PES (XXXIII, 2); NOX (XXXIII, 64; 189; 477); SOL (XXXIII, 264); VIS (XXXIII, 57). 
sula de carácter ornamental aparecen ante nuestros ojos como hechos indiscutibles.

Catulo, por el contrario, no presenta este tipo conformado con las mismas caracteristicas. Prefiere cerrar el verso con formas pronominales - aunque inserte MORS, la fatídica que se llevó a su hermano-y resaltar con este final anómalo la persona, casi siempre protagonista en el poema, a que se refiere. Parece, pues, evidente que su disposición se distancia de la ciceroniana, salvo en el deseo de situar en relevancia el término monosilábico.

En época augústea, el uso del monosílabo final aislado muestra las frecuencias siguientes, según los autores:

\begin{tabular}{|c|c|c|c|}
\hline VIRGILIO & HORACIO TIBULO & PROPERCIO & OVIDIO \\
\hline $\begin{array}{l}47 \\
0.36 \%\end{array}$ & $\begin{array}{l}176 \\
4,18 \%\end{array}$ & & $\begin{array}{l}12 \\
0,05\end{array}$ \\
\hline
\end{tabular}

Será Virgilio, de nuevo, quien ofrezca una muy precisa caracterización de este tipo clausular. De los datos que hemos podido recoger, se desprende que el mantuano propone un monosílabo final aislado - que configuraría una cláusula anómala tanto desde el punto de vista tipológico como acentual-, sólo cuando utiliza términos plenos desde el punto de vista semántico: son vocablos sonoros, a veces con resonancias de poetas anteriores - sobre todo de Ennio- que se acompañan de otros recursos, generalmente fonoestilísticos, para que adquieran mayor relieve.

Por el contrario, cuando el último semipié está ocupado por un término gramatical, el poeta lo hace preceder de otro monosílabo con el que puede formar «mot-métrique», de modo que la cláusula aparece cerrada con el esperado «bisílabo», y dentro de los cauces de la normalidad.

Detallaremos los lugares correspondientes en las Geórgicas - para no abrumar con los datos correspondientes a la totalidad de los casi 13.000 versos que nos ofrece la obra virgiliana-. En la citada obra encontramos los siguientes versos finalizados en un monosílabo gramatical:

\footnotetext{
- 3 ET CVM (I, 313; I, 370; III, 133)

- 2 SI QVIS (II, 49; III, 474)

- 1 QVAE SINT (II, 103)

- 1 ET DVM (III, 428)

- 1 IN SE (III, 484)

- 1 SI QVEM (IV, 6)

- 1 AVT HOS (IV, 84)
} 
Por el contrario, cuando Virgilio cierra el verso con un término pleno ofrece las disposiciones siguientes:

- EXIGVVS MVS $(I, 181)$

- SILET NOX (I, 247)

- IMBRIFERVM VER- (I, 313)

- RAPIDVS SOL (II, 321)

- EXACVIT SVS (III, 255)

- INCREPAT ET VOX (IV, 71).

Este último ejemplo, a pesar de que finaliza en doble monosilabo debería incluirse entre los anómalos, primero, porque acentualmente lo sería, y segundo, porque cumple otras condiciones habituales en las cláusulas monosilábicas, que ahora señalaremos.

Tras la consideración de estas seis cláusulas, fácilmente se percibe que aparecen formadas por el conjunto epiteto-sustantivo o verbo-sujeto, modelando una unidad que ofrece una cierta autonomía en el conjunto del verso. Por otra parte se puede apreciar otros fenómenos como el homeoteleuto (EXIGVVS MVS) o la paréchesis, presente en cada uno de estos finales (y señalada previamente en los textos que transcribimos), generalmente con repetición del sonido silbante $\mathrm{y}$, en otros casos, de la vibrante -r- y de los sonidos - V- y -K-.

Otra característica a añadir sería la evocación de algunos finales de Ennio: así, CVM RAPIDVS SOL recogería EXORITVR SOL $(A n$. I, 96), o INCREPAT ET VOX la secuencia OBSTITIT ET NOX $(A n$. IV, 166$)$.

Esta circunstancia se hace aún más evidente en otros finales de la Eneida, como en el verso 12 del libro III donde ET MAGNIS DIS reproduce casi exactamente CVM MAGNIS DIS de An. VI, 193; o en En. XII, 552, cuya secuencia NITVNTVR OPVM VI repite punto por punto la que aparece en $A n$. XVI, 394.

Según estas consideraciones parece que dos rasgos son fundamentales a la hora de definir la cláusula monosilábica en Virgilio, se trataría de un final ornamental y arcaizante.

La situación en Horario es completamente diversa y marca una clara diferencia entre la factura de los versos más líricos, los didácticos y los satíricos.

Es cierto que el monosílabo final aislado aparece en las Odas y los Épodos con unos porcentajes elevados, que sin duda vienen forzados por el escaso número de versos que se puede reunir; sin embargo, en las cuatro cláusulas que hemos recogido sólo dos responden a la fórmula virgiliana: VNA MANET NOX (Odas I, 18,15) y AVT MELIOR PARS (Ep. 16,15 ), con un término sonoro como broche del verso y paréchesis, más acentuada en el primer caso. En los otros dos ejemplos CONVIVA, MAGIS 
QVEM (Ep. 12,23) y ATQVE MEO NVNC ( $E p .15,17)$, el último presenta un término gramatical que, sin embargo, ni puede formar mot-métrique con el anterior, ni parece en especial relevancia.

Si en las Odas y Épodos el promedio de cláusulas monosilábicas que recogian los modos del mantuano ascendian al $50 \%$, en las Epistolas desciende al $41,26 \%$ y en las Sátiras al $24,76 \%$.

La cláusula con monosílabo final ornamental sólo tiene una incidencia media en la obra lírica del poeta, mientras que pierde refrendo en los versos satíricos, dejando paso a un tipo clausular en que el término gramatical de una sola silaba - como señaló ya Brink ${ }^{21}$ - se utiliza para facilitar el encabalgamiento de dos versos consecutivos. Función semejante tienen, en muchas ocasiones, los abundantes finales - tanto en las Sátiras como en las Epístolas - construidos con dos monosílabos.

Pero, con respecto al gusto de Horacio por estos remates monosilábicos, debemos avanzar aún un poco más, hasta señalar un tipo de cláusula caracterizada por la presencia de tres monosílabos (ya sean términos plenos o por elisión) que provocan un final entrecortado, cuyo objeto sería quitar solemnidad a la cláusula hexamétrica y acentuar su carácter conversacional. Asimilados a éstos estarían los finales con la estructura $1+2+1+1$ (o las similares en que el longum $\mathrm{V}$ estuviera ocupado por la sílaba larga final de un polisilabo), cuya sec sencia ofrece igualmente una cláusula dividida.

En este sentido, nuestro recuento apoya claramente lo dicho, ya que ni en las Odas ni en los Épodos aparece un final con tales características. Por otra parte, en las Epistolas I y II obtenemos un porcentaje de $1,18 \%$ y $1,25 \%$, respectivamente, mientras que en el Ars -más próximo también en otros detalles a la factura virgiliana - ha descendido al $0,83 \%$.

En las Sátiras, por el contrario, el promedio asciende a $1,98 \%$, registrándose además un gran número de elisiones, incluso dos en una misma cláusula, a diferencia de lo que ocurre en el hexámetro dactílico épico o lírico.

Parece, pues, oportuno, suponer que Horacio ha utilizado -en el hexámetro satírico fundamentalmente - un tipo de cláusula caracterizado por la presencia de al menos dos monosílabos - preferentemente por tres, y con el concurso de la elisión-, que tiene por objeto restar formalismo a la construcción de estos finales, acercándolos hacia una expresión más propia de la lengua familiar.

Tibulo - como resulta lo esperado dada la trayectoria que nos viene ofreciendo- no hace uso de este final monosilábico, y sólo en una ocasión elige el concurso de dos monosílabos formando "mot-métrique" para concluir uno de sus hexámetros.

"I Brink, C. O.: O.c., p. 226. 
En Propèrcio este tipo de cláusula presenta también carácter excepcional. En este caso el poeta no se muestra seguidor del mantuano, ya que su hexámetro (II, XXV, 17) termina con un pronombre relativo cuya función es introducir un encabalgamiento que vincule estrechamente las dos líneas del dístico.

Las razones que Propercio haya tenido para menospreciar este final no son patentes, pero acudiremos a las que más tarde comentaremos para Ovidio: su carácter arcaizante; su carácter heterodínico, y su especial vinculación a la épica.

El poeta de Sulmona utiliza también con parquedad este final hexamétrico, pues los 12 ejemplos que hemos registrado suponen un promedio del $0,05 \%$.

Resulta sorprendente, sin duda, comprobar cómo el autor prescinde absolutamente del monosílabo final aislado hasta bien avanzada su obra, concretamente hasta el libro V de las Metamorfosis.

La razón de este comportamiento podríamos buscarla en el rechazo del carácter arcaico que se puede atribuir a este final, pero -quizá con una mayor garantía - podría también aludirse a su realización claramente heterodínica.

Efectivamente, a partir del uso ovidiano de las cláusulas con un final en jónico «a minore», hemos podido percibir su sensibilidad hacia la cuestión acentual: Ovidio utiliza en estos finales sólo la distribución con heterodinia -dado su carácter helenizante-, probablemente en la idea de que la homodinia era un rasgo específicamente romano y no adecuado, por tanto, en este conjunto.

La situación del acento en la cláusula debía de presentar para Ovidio unas connotaciones diversas de las que el mantuano podía percibir. Seguramente ahora el valor de la homodinia en la cláusula habia tomado cuerpo en la rítmica del verso, aun sin haber llegado a ser algo imprescindible.

Desde esta perspectiva podemos entender que Ovidio no llegara a utilizar el monosílabo aislado hasta su obra más estrictamente épica, reflejando además en la mayor parte de los casos las disposiciones virgilianas. A este respecto conviene anotar que los Tristia presentan un solo ejemplo de esta cláusula, mientras que los Pontica - más próximos a la factura del mantuano también por otras razones- ofrece tres monosílabos aislados como broche del verso.

Los finales que encontramos en las Metamorfosis son los siguientes:

$-\mathrm{V}, 573$

- VII, 520

- VII, 663

- VIII, 359

- VIII, 603

- XIV, 515
ARETHVSA, SACER FONS

AMBAGE MŌRER VOS

EXTVLERAT SOL

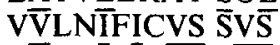

AEQVŌ $\bar{E} \bar{V} \underline{\mathrm{S}}$ REX

SEMICAPER $\underline{\mathrm{PAN}}$ 
Salvo en el verso VII, 520 que prescinde del enfoque "virgiliano", en los demás casos podemos decir que, de una manera más o menos ajustada, se respetan las normas que hemos observado en Virgilio: sujeto;

$1 .^{\circ}$, la cláusula se construye con el binomio epiteto-sustantivo o verbo-

$2 .^{\circ}$, aparecen términos plenos y sonoros como broche del verso;

$3 .^{\circ}$ las cláusulas se adornan con recursos de carácter fono-estilístico, fundamentalmente la paréchesis o el homeoteleuto.

En los versos elegiacos, sin embargo, sólo uno de los ejemplos se ajusta a estas caracteristicas, se trata de Pont. IV, 2,47 con el final AONIVS FONS.

En cuanto a Tris. II, 433 (CARMEN, APVD QVOS) y Pont. I, 3,81 (GENTIS APVD QVOS), podrían resultar evocación de dos finales de corte similar recogidos en las obras de Catulo y Virgilio: COPIA APVD ME ( $C$. 68,33) y SEMPER APVD ME (Buc. III, 62), respectivamente.

El último ejemplo, sin embargo, cae fuera de las normas de la «imitatio»; se trata de Pont. IV, 9,101, con la fórmula DECRETA QVIBVS NOS.

4. Con respecto a la última de las cláusulas que vamos a considerar, el tipo denominado ARMAQVE NOSTRA, conviene subrayar que en este caso la anomalia no estaba en la dificultad tipológica, sino en la acentuación. En efecto, al situarse el acento de la palabra sobre la primera breve del bíceps $\mathrm{V}$ - en lugar de sobre el longum correspondiente-, se produce una ruptura tocante al orden esperado, de tal manera que un verso finalizado con tal disposición, si bien no puede ser tachado de defectuoso, tiene que considerarse a la fuerza «diferente» de los habituales.

El uso de esta cláusula en los poetas más antiguos fue como sigue:

\begin{tabular}{ccccc} 
& ENNIO & LUCILIO & LUCRECIO \\
\cline { 2 - 4 } ARMAQVE NOSTRA & $\ldots \ldots . .$. & 4 & 1 & 100 \\
& $0,69 \%$ & $0,21 \%$ & 1,34
\end{tabular}

En el autor de los Anales la frecuencia de este tipo no alcanza un promedio elevado y, desde luego, se presenta muy por debajo de la de los anómalos buscados y queridos por él, como los tipos EXORITVR SOL $(5,66 \%)$ o QVADRVPEDANTVM $(3,65 \%)$.

Es muy posible que, dada la técnica de elaboración del verso enniano 
descrita por Cordier ${ }^{22}$, este grupo verbal con la enclítica, al situarse en el final de verso, habría presentado el problema de aparejar otro grupo verbal antes o después con el que efectuar la coordinación, lo que hubiera podido provocar algunas dificultades. Además este tipo de final no resultaba relevante dentro de las estructuras griegas, por lo que no era especialmente deseable.

Entre los cuatro ejemplos detectados en los Anales, dos presentan encabalgamiento, otro se produce ante pausa fuerte y el cuarto ha perdido su continuación.

Podemos, con una cierta seguridad, suponer que en Ennio la estructura con enclítica presenta encabalgamiento en un $50 \%$ de los casos; ello estaría de acuerdo con el estudio de Soubiran ${ }^{23}$, según el cual, entre las estructuras con enclítica, la mitad se construye habitualmente con grupos amplios que, en final de verso, abocarían necesariamente al encabalgamiento.

En cuanto a Lucilio, el uso de la cláusula ARMAQVE NOSTRA aparece claramente restringido: encontramos un solo ejemplo, CEPHALAEAQVE ACARNAE (Sát. I, 25,2), es un final de fragmento, por lo que no sabemos con exactitud si continuaría en el verso siguiente; a primera vista, sin embargo, el sentido parece completo.

Evidentemente, este tipo clausular no resultaba oportuno en la sátira de Lucilio, bien fuera por su carácter homodínico, bien por la supuesta vinculación de la enclítica - que al género épico, o por ambas cosas a la vez.

Lucrecio, por el contrario, utiliza con largueza esta distribución, alcanzando un promedio superior al que será modélico según los usos virgilianos. Sin embargo, su empleo no presenta ningún rasgo especialmente representativo, ya que el encabalgamiento aparece en un $50 \%$ de los $\operatorname{casos}^{24}$-como sería norma- y el autor no la utiliza para sus esquemas repetitivos, tan oportunos en el final del verso. Podríamos, pues, concluir que la cláusula ARMAQVE NOSTRA pasó por la obra lucreciana con algo más gloria que pena (por su porcentaje), pero de manera un tanto anodina.

Con respecto al período que hemos llamado de extensión, los promedios de uso fueron los siguientes:

\begin{tabular}{|c|c|c|}
\hline & CATULO & CICERÓN \\
\hline ARMAQVE NOSTRA ....... & $\begin{array}{c}1 \\
0,13 \%\end{array}$ & $\begin{array}{c}2 \\
0,27 \%\end{array}$ \\
\hline
\end{tabular}

${ }^{22}$ Cordier, A.: O.c., p. 15.

2.3 Soubiran, J.: «Sur les mots de type armaque dans l'hexamètre latin», Pallas 14, 1967, pp. 42 ss.

${ }^{24}$ Arribas, M. L.: O.c., p. 202. 
En Catulo el único ejemplo que hemos registrado aparece en C. LXIV, 348 , donde el sintagma complemento directo queda en suspenso hasta la emisión del conjunto verbo-sujeto en el verso 349.

Evidentemente, Catulo ha querido vincular este tipo de cláusula a una expresión de especial relevancia, como resultan generalmente aquellos finales suyos que aparecen en posición de «rejet» o encabalgamiento, según pudo comprobar Cupaiuolo ${ }^{25}$.

Cicerón, por su parte, entre los versos recibidos, ha querido utilizar este tipo en dos ocasiones, una en su obra de juventud (Arat. XXXIII, 2: STELLAQVE IVNGENS) y otra en la madurez (De consul. 32: VENERATAQVE NATTAE), en ambos casos vinculado al encabalgamiento.

No podemos decir, realmente, que en este periodo el tipo ARMAQVE NOSTRA aparezca como estructura clausular viva y vigorosa, pero lo cierto es que se muestra ya de alguna manera caracterizado, pues en un 100 por 100 de los casos aparece unido al encabalgamiento.

En época augústea, sin embargo, el panorama se altera en alguna medida, ya que el tipo ARMAQVE NOSTRA recibe una gran acogida por parte de Virgilio, aunque se utiliza sólo moderadamente por parte de Horacio y Ovidio. Su promedio en los distintos autores es el siguiente:

\section{ARMAQVE NOSTRA.}

\begin{tabular}{ccccc} 
VIRGILIO & HORACIO & TIBULO & PROPERCIO & OVIDIO \\
\hline 130 & 19 & - & 2 & 105 \\
$1,01 \%$ & $0,45 \%$ & & $0,10 \%$ & $0,45 \%$
\end{tabular}

Virgilio, en su primera obra atribuida, ha empleado esta distribución sólo en un verso que no se prolonga en encabalgamiento; por el contrario, en las Geórgicas alcanza el promedio más elevado (1,30\%), por encima incluso del de la Eneida $(1,08 \%)$. Entre los 26 versos que la presentan, 18 rompen la pausa versal, con lo que el promedio de encabalgamientos es un $69,23 \%$, bastante por encima del $50 \%$ esperado.

En la Eneida, la vinculación de este tipo con el encabalgamiento se hace aún más estrecha, pues el porcentaje de versos que lo presenta alcanza un $73,78 \%$. Evidentemente, la cifra supera demasiado el promedio esperado, como para pensar que su ascenso es fruto del azar.

Muy atinadas a este respecto resultan unas palabras de Soubiran que reparó ya en este detalle, y lo puso de relieve en sus artículos acerca de los tipos ARMAQVE y ARMENTAQVE ${ }^{26}$, cuando refiriéndose a ellos afirma:

${ }^{25}$ Cupaiuolo, F.: Studi sull'esametro di Catullo, Nápoles 1965, p. 29.

26 Soubiran, J.: O.c. y "Sur les mots de type Armentaque dans l'hexamètre latin", Pallas 15, 1968, pp. 57-101. 
«l'on peut dire que leur caractère est d'attirer l'attention sur le vers qui suit».

Y para «atraer la atención» del lector en esta zona específica del verso que comprende la cláusula y el comienzo del verso siguiente, Virgilio se ha servido en el nivel estilístico, del encabalgamiento, y en el nivel métrico, de la heterodinia; ha sabido combinar en un solo final típico dos claras desviaciones de la norma: la ruptura de la pausa versal y la ruptura de la secuencia acentual esperada.

Este tipo clausular manifiestamente caracterizado por Virgilio desde el punto de vista estilístico, y de gran vigencia en su obra, será imitado con posterioridad, penetrando incluso en la Edad Media, como se desprende de nuestro estudio anteriormente citado.

En cuanto a Horacio, su trayectoria en el uso de estos tipos refleja la misma distribución que hemos debido dibujar con respecto a las cláusulas ya estudiadas.

La fórmula ARMAQVE NOSTRA — vinculada al encabalgamiento en los ejemplos que nos provee el hexámetro más lírico y en un buen número de casos en las Epistolas - ofrece un promedio elevado en los Epodos y en las Odas de la primera época, (de factura más cercana al hexámetro virgiliano), disminuye en las Epistolas (más próximas al lenguaje conversacional), y encuentra su cota más baja $(0,23 \%)$ en las Sátiras - como sería lo esperado-, dado que este tipo clausular se presenta como una fórmula claramente estilizada.

A este respecto, conviene señalar cómo Horacio en las Sátiras ha querido alejarse en alguna manera de la técnica virgiliana, primero en el aspecto formal, porque, entre los cinco ejemplos que hemos registrado, uno utiliza ante la enclítica un final en $-e(I, 1,89)$, frente a la acostumbrada desinencia en $-a$. Además en dos versos $(\mathrm{I}, 1,89$ y II, 8,76$)$ se respeta claramente la pausa versal y en $I, 2,73$, se podría apreciar un encabalgamiento laxo, puesto que los términos que integran la cláusula forman parte de una frase que prosigue en el verso siguiente, pero no son ya ellos mismos los vehículos de la continuidad. La ruptura clara de la pausa versal en la cláusula se encontraría sólo en dos finales: II, 3, 95, HVMANAQVE PVLCHRIS // DIVITIIS y II, 6, 85, SEMESSAQVE LARDI // FRVSTA.

Tibulo continúa transitando los mismos senderos que ya señalamos anteriormente, pues prescinde absolutamente del uso de la cláusula tipo ARMAQVE NOSTRA.

En cuanto a Propercio, utiliza el tipo con una cierta reserva aunque, cuando lo hace, parece seguir los dictámenes del mantuano, ya que, de los dos casos en que lo aprovecha, en uno (II, XXVI, 31) lo hace con encabalgamiento estricto y en otro (III, XX, 15) con encabalgamiento laxo.

Cabe de nuevo preguntarse si el elegíaco utiliza en tan escasa medida esta distribución porque había quedado configurada - a partir del uso vir- 
giliano- como algo característico de la épica, o bien porque Propercio prefería respetar la consabida secuencia acentual que situaba el acento de la palabra sobre los longa $\mathrm{V}$ y VI.

Ovidio se sirve de esta cláusula con una trayectoria que nos resulta curiosa, pero cuyas razones parecen asequibles.

El autor requiere la distribución ARMAQVE NOSTRA en su primera época con un entusiasmo que va decreciendo con el paso del tiempo, pues en las primeras Heroidas (20-16 a. J.C.) y los Amores (15 a. J.C.) la proporción es de $0,55 \%$ y $0,79 \%$ respectivamente, mientras que en el Ars (1 a. J.C.) ésta ha descendido hasta un 0,34\%, desapareciendo en De Medicamine, Remedia y las últimas Heroidas, cuya creación discurre entre los años 1 y 8 d. J.C.)

En los Fastos (8 d. J.C.), seguramente por su carácter más próximo a la épica, nuestra cláusula vuelve a aparecer $(0,44 \%)$, y aumenta su frecuencia sobre todo en las Metamorfosis $(0,63 \%)$, para desaparecer en los Tristia y renacer en los Pontica, obra más virgiliana también por otros conceptos. Inusitada en el $\mathbf{I b i s}$, presenta un solo ejemplo en el poema didáctico que se le atribuye por algunos autores, Helieutica.

La factura de esta cláusula en Ovidio no dista de la virgiliana, pues se utiliza igualmente para introducir el encabalgamiento en un $86,09 \%$ de sus versos, proporción que supera incluso el $73,08 \%$ del mantuano.

Así pues, Ovidio debió de utilizar este tipo de finales, en su primera época en parte como epígono de Virgilio, en parte por la facilidad que le otorgaba para realizar la deseada vinculación entre las dos líneas del dístico elegíaco. En un momento de su obra, sin embargo, parece rechazarlo quizá en virtud de su heterodinia. Finalmente, es acogido de nuevo, casi con seguridad en razón de su afinidad con el género épico y, quizá también en cierta medida, como tributo de admiración hacia el autor de la Eneida.

II. De acuerdo con el detenido análisis que presentamos, hemos podido obtener las siguientes conclusiones:

1. Las cláusulas denominadas «anómalas» ya desde las primeras manifestaciones del hexámetro dactílico muestran una cierta especialización, desde el punto de vista de la expresión de estilo.

2. Es Virgilio, precisamente, el autor que recoge todos los valores estilísticos que se habían ido insinuando en poetas anteriores, creando una especie de sistema en que a cada una de las cláusulas no convencionales le corresponde una matización estilística concreta.

3. Tibulo es el único poeta que prescinde de las cláusulas anómalas recurriendo casi absolutamente al empleo de los tres tipos «acostumbrados». 
4. Los v̀alores estilísticos en estas cláusulas extraordinarias nos dan ocasión para otorgarles una denominación, que sirve a la par para individualizarlas y para su clasificación. Nuestra denominación es la siguiente:

Cláusulas helenizantes: son los tipos recogidos bajo los lemas QVADRVPEDANTVM, DI GENVERVNT y GEMITV LACRIMISQVE; es decir, las que se construyen con un pentasílabo o con un tetrasílabo en forma de jónico «a minore». Generalmente aparecen ocupadas con nombres helénicos y producen —en los tipos con tetrasílabo - fenómenos prosódicos inhabituales en la lengua latina, como el alargamiento en arsis, el hiato o el mantenimiento de la vocal larga ante la inicial vocálica subsecuente.

Cláusulas neotéricas: se recogen bajo el lema APPENNINOS y están constituidas generalmente por un tetrasílabo con estructura dispondaica o, en su defecto, por un moloso más la última sílaba larga del término precedente.

Dado que representan la evocación de un tipo ya especialmente caracterizado en la métrica griega, no resulta extraño que en ocasiones quiera adornarse con los rasgos prosódicos helénicos que antes mencionamos, y que, con alguna frecuencia, elija nombres de raigambre griega, sin que este detalle se repita de modo sistemático, como ocurría en el tipo anteriormente considerado.

Cláusulas ornamentales: quedan acogidas bajo el lema EXORITVR SOL y se construyen con un monosílabo final precedido de un bisílabo o polisílabo. Estos monosílabos son nombres sonoros, de significado pleno, que en el broche del verso aparecen en situación relevante.

El final hexamétrico que presenta esta distribución suele recoger un sintagma en cierta manera independiente, integrado por el conjunto epiteto-sustantivo o verbo-sujeto.

En otro sentido, estas cláusulas aparecen también caracterizadas en Virgilio por una aureola de arcaísmo, ya que un cierto número de ellas parece evocación clara de otras utilizadas por Ennio en sus Anales, poema donde este tipo clausular alcanza la más alta frecuencia de uso, en comparación con las restantes cláusulas extraordinarias.

Cláusulas satíricas. No aparecen -como es natural- estos tipos en la órbita virgiliana, sino de la mano de Horacio, que recoge una distribución existente ya en Lucilio, aunque poco utilizada. Su es- 
quema se realiza generalmente por las secuencias $1+2+1+1$ ó $2+1+1+1$. Su finalidad consistiría en presentar una cláusula entrecortada que quitara solemnidad al final hexamétrico y le hiciera próximo a la lengua coloquial. Para este efecto, el poeta hace frecuente recurso a la elisión.

Cláusulas encabalgantes: se pueden algutinar bajo la denominación ARMAQVE NOSTRA, y se realizan cuando al bisílabo final le precede un término unido a la partícula enclítica; bajo esa situación, el acento de palabra recae en una de las sílabas breves del quinto pie, de manera que el poeta - aun manteniendo la tipología más regular- debe renunciar a la consabida homodinia.

Virgilio —el autor que más la autilizó en época augústea y que supo conformarla para la posteridad-se sirvió de este tipo como vehículo adecaudo para romper la pausa versal. Su diseño fue compartido por los poetas contemporáneos y, posteriormente, alcanzó un gran desarrollo, especialmente en la época argéntea. 